\title{
Composition Formulas of Bessel-Struve Kernel Function
}

\author{
K. S. Nisar, ${ }^{1}$ S. R. Mondal, ${ }^{2}$ and P. Agarwal ${ }^{3}$ \\ ${ }^{1}$ Department of Mathematics, College of Arts and Science, Prince Sattam Bin Abdulaziz University, P.O. Box 54, \\ Wadi Al-Dawasir 11991, Saudi Arabia \\ ${ }^{2}$ Department of Mathematics \& Statistics, College of Science, King Faisal University, P.O. Box 400, \\ Hofuf, Al-Ahsa 31982, Saudi Arabia \\ ${ }^{3}$ Department of Mathematics, Anand International College of Engineering, Jaipur, India \\ Correspondence should be addressed to P. Agarwal; goyal.praveen2011@gmail.com
}

Received 30 December 2015; Accepted 24 February 2016

Academic Editor: José A. T. Machado

Copyright (c) 2016 K. S. Nisar et al. This is an open access article distributed under the Creative Commons Attribution License, which permits unrestricted use, distribution, and reproduction in any medium, provided the original work is properly cited.

\begin{abstract}
The object of this paper is to study and develop the generalized fractional calculus operators involving Appell's function $F_{3}(\cdot)$ due to Marichev-Saigo-Maeda. Here, we establish the generalized fractional calculus formulas involving Bessel-Struve kernel function $S_{\alpha}(\lambda z), \lambda, z \in \mathbb{C}$ to obtain the results in terms of generalized Wright functions. The representations of Bessel-Struve kernel function in terms of exponential function and its relation with Bessel and Struve function are also discussed. The pathway integral representations of Bessel-Struve kernel function are also given in this study.
\end{abstract}

\section{Introduction}

Fractional calculus has found applications in various and extensive fields of engineering and science such as electromagnetics, fluid mechanics, signals processing, and control theory. It has been used to model physical and engineering processes that are found to be best described by fractional differential equations. Recent researches observed that the solutions of fractional-order differential equations could model real-life situations better, particularly in reaction-diffusiontype problems. Due to the potential applicability to a wide variety of problems, fractional calculus is developed to a large area of mathematics and other engineering applications [14]. The fractional integral operator involving several special functions has found great importance and applications in many subfields such as statistical distribution theory, control theory, fluid dynamics, stochastic dynamical system, nonlinear biological systems, astrophysics, and quantum mechanics (see [5-7]).

The influence of fractional integral operators involving various special functions in fractional calculus is very important due to its significance and applications in various subfields of applied mathematical analysis. Many studies related to the fractional calculus are found in the papers of
Love [8], McBride [9], Agarwal and Nieto [10], Kalla [11], Kalla and Saxena [12, 13], Saigo [14-16], Saigo and Maeda [17], and Kiryakova [18]. A comprehensive explanation of such operators is given by Miller and Ross [19] and Kiryakova [18].

Recently, researchers investigated and studied about the fractional integration formulas for the Bessel function and generalized Bessel functions (see [20, 21]). The generalization of Bessel function and its applications in fractional transforms are found in $[22,23]$. A useful generalization of the hypergeometric fractional integrals, including the Saigo operators ([14-16]), has been introduced by Marichev [24] (see details in Samko et al. [25, page 194]) and later extended and studied by Saigo and Maeda ([17, page 393]) in terms of any complex order with Appell function $F_{3}(\cdot)$ in the kernel, as follows.

Let $\alpha, \alpha^{\prime}, \beta, \beta^{\prime}, \gamma \in \mathbb{C}$ and $x>0$; then the generalized fractional calculus operators involving the Appell function are defined as follows:

$$
\begin{aligned}
& \left(I_{0,+}^{\alpha, \alpha^{\prime}, \beta, \beta^{\prime}, \gamma} f\right)(x)=\frac{x^{-\alpha}}{\Gamma(\gamma)} \int_{0}^{x}(x-t)^{\gamma-1} \\
& \cdot t^{-\alpha^{\prime}} F_{3}\left(\alpha, \alpha^{\prime}, \beta, \beta^{\prime} ; \gamma ; 1-\frac{t}{x}, 1-\frac{x}{t}\right) f(t) d t,
\end{aligned}
$$




$$
\begin{aligned}
& \left(I_{0,-}^{\alpha, \alpha^{\prime}, \beta, \beta^{\prime}, \gamma} f\right)(x)=\frac{x^{-\alpha^{\prime}}}{\Gamma(\gamma)} \int_{x}^{\infty}(t-x)^{\gamma-1} \\
& \cdot t^{-\alpha} F_{3}\left(\alpha, \alpha^{\prime}, \beta, \beta^{\prime} ; \gamma ; 1-\frac{t}{x}, 1-\frac{x}{t}\right) f(t) d t,
\end{aligned}
$$

with $\operatorname{Re}(\gamma)>0$. For more details about the above operators, see $[17,24]$. The generalized fractional integral operators of the type (1) and (2) have been introduced by Marichev [24] and later extended and studied by Saigo and Maeda [17] (this operator known as the Marichev-Saigo-Maeda operator). For the definition of the Appell function $F_{3}\left({ }^{\circ}.\right)$ the interested readers may refer to the monograph by Srivastava and Karlson [26] (see also Erdélyi et al. [27] and Prudnikov et al. [28]). The applications of fractional integral operators are found in many papers $([19,22,29-31])$. The following results given in $[17,32]$ are needed in sequel.

Lemma 1. Let $\alpha, \alpha^{\prime}, \beta, \beta^{\prime}, \gamma, \rho \in \mathbb{C}$ such that $\operatorname{Re}(\gamma)>0$ and

$$
\operatorname{Re}(\rho)>\max \left\{0, \operatorname{Re}\left(\alpha-\alpha^{\prime}-\beta-\gamma\right), \operatorname{Re}\left(\alpha^{\prime}-\beta^{\prime}\right)\right\} .
$$

Then there exists the relation

$$
\begin{aligned}
& \left(I_{0,+}^{\alpha, \alpha^{\prime}, \beta, \beta^{\prime}, \gamma} t^{\rho-1}\right)(x) \\
& =\Gamma\left[\begin{array}{c}
\rho, \rho+\gamma-\alpha-\alpha^{\prime}-\beta, \rho+\beta^{\prime}-\alpha^{\prime} \\
\rho+\beta^{\prime}, \rho+\gamma-\alpha-\alpha^{\prime}, \rho+\gamma-\alpha^{\prime}-\beta
\end{array}\right] x^{\rho-\alpha-\alpha^{\prime}+\gamma-1},
\end{aligned}
$$

where

$$
\Gamma\left[\begin{array}{l}
a, b, c \\
d, e, f
\end{array}\right]=\frac{\Gamma(a) \Gamma(b) \Gamma(c)}{\Gamma(d) \Gamma(e) \Gamma(f)}
$$

Lemma 2. Let $\alpha, \alpha^{\prime}, \beta, \beta^{\prime}, \gamma, \rho \in \mathbb{C}$ such that $\operatorname{Re}(\gamma)>0$ and

$$
\begin{aligned}
& \operatorname{Re}(\rho)<1 \\
& \quad+\min \left\{\operatorname{Re}(-\beta), \operatorname{Re}\left(\alpha+\alpha^{\prime}-\gamma\right), \operatorname{Re}\left(\alpha+\beta^{\prime}-\gamma\right)\right\} .
\end{aligned}
$$

Then there exists the relation

$$
\left(I_{0,-}^{\alpha, \alpha^{\prime}, \beta, \beta^{\prime}, \gamma} t^{\rho-1}\right)(x)=\Gamma\left[\begin{array}{c}
1-\rho-\gamma+\alpha+\alpha^{\prime}, 1-\rho+\alpha+\beta^{\prime}, 1-\rho-\beta \\
1-\rho, 1-\rho+\alpha+\alpha^{\prime}+\beta+\beta^{\prime}-\gamma, 1-\rho+\alpha-\beta
\end{array}\right] x^{\rho-\alpha-\alpha^{\prime}+\gamma-1}
$$

The generalized Wright hypergeometric function ${ }_{p} \psi_{q}(z)$ is defined by the series

$$
\begin{aligned}
{ }_{p} \psi_{q}(z) & ={ }_{p} \psi_{q}\left[\begin{array}{c}
\left(a_{i}, \alpha_{i}\right)_{1, p} \\
\left(b_{j}, \beta_{j}\right)_{1, q}
\end{array} \mid z\right] \\
& =\sum_{k=0}^{\infty} \frac{\prod_{i=1}^{p} \Gamma\left(a_{i}+\alpha_{i} k\right)}{\prod_{j=1}^{q} \Gamma\left(b_{j}+\beta_{j} k\right)} \frac{z^{k}}{k !} .
\end{aligned}
$$

Here $a_{i}, b_{j} \in \mathbb{C}$, and $\alpha_{i}, \beta_{j} \in \mathbb{R}(i=1,2, \ldots, p ; j=$ $1,2, \ldots, q)$. Asymptotic behavior of this function for large values of argument of $z \in \mathbb{C}$ was studied in [33] and under the condition

$$
\sum_{j=1}^{q} \beta_{j}-\sum_{i=1}^{p} \alpha_{i}>-1
$$

in [34-38]. The Bessel and modified Bessel functions of first kind, the Struve function $H_{v}(z)$, and modified Struve function $L_{v}(z)$ possess power series representation of the form [39]

$$
\begin{aligned}
& J_{v}(z)=\sum_{k=0}^{\infty} \frac{(-1)^{k}(z / 2)^{2 k+v}}{\Gamma(k+v+1) k !}, \\
& I_{v}(z)=\sum_{k=0}^{\infty} \frac{(z / 2)^{2 k+v}}{\Gamma(k+v+1) k !},
\end{aligned}
$$

$$
\begin{aligned}
& H_{v}(z) \\
& \quad=\left(\frac{z}{2}\right)^{v+1} \sum_{k=0}^{\infty} \frac{(-1)^{k}}{\Gamma(k+3 / 2) \Gamma(k+v+1 / 2)}\left(\frac{z}{2}\right)^{2 k}, \\
& L_{v}(z) \\
& \quad=\left(\frac{z}{2}\right)^{v+1} \sum_{k=0}^{\infty} \frac{1}{\Gamma(k+3 / 2) \Gamma(k+v+1 / 2)}\left(\frac{z}{2}\right)^{2 k} .
\end{aligned}
$$

The Bessel-Struve kernel $S_{\alpha}(\lambda z), \lambda \in \mathbb{C}$, [40] which is unique solution of the initial value problem $l_{\alpha} u(z)=$ $\lambda^{2} u(z)$ with the initial conditions $u(0)=1$ and $u^{\prime}(0)=$ $\lambda \Gamma(\alpha+1) /(\sqrt{\pi} \Gamma(\alpha+3 / 2))$, is given by $S_{\alpha}(\lambda z)=j_{\alpha}(i \lambda z)-$ $i h_{\alpha}(i \lambda z), \forall z \in C$, where $j_{\alpha}$ and $h_{\alpha}$ are the normalized Bessel and Struve functions.

Moreover, the Bessel-Struve kernel is a holomorphic function on $\mathbb{C} \times \mathbb{C}$ and it can be expanded in a power series in the form

$$
S_{\alpha}(\lambda z)=\sum_{n=0}^{\infty} \frac{(\lambda z)^{n} \Gamma(\alpha+1) \Gamma((n+1) / 2)}{\sqrt{\pi} n ! \Gamma(n / 2+\alpha+1)} .
$$

The present paper is organized as follows. The composition of integral transforms (1) and (2) with BesselStruve kernel function and the relation between BesselStruve function and other functions are given in Section 2. Section 3 investigates the pathway fractional integration of the Bessel-Struve kernel function and finally the concluding remark is drawn in Section 4. 


\section{Fractional Integral Formulas}

In this section we will investigate the composition of integral transforms (1) and (2) with the Bessel-Struve kernel function defined in (11).
Theorem 3. Let $\alpha, \alpha^{\prime}, \beta, \beta^{\prime}, \gamma, \rho, p, b, c, \lambda, \nu \in \mathbb{C}$. Suppose that $\operatorname{Re}(\gamma)>0$ and $\operatorname{Re}(\rho+n)>\max \left\{0, \operatorname{Re}\left(\alpha+\alpha^{\prime}+\beta-\gamma\right), \operatorname{Re}\left(\alpha^{\prime}-\right.\right.$ $\left.\beta^{\prime}\right)$ \}. Then

$$
\begin{aligned}
\left(I_{0,+}^{\alpha, \alpha^{\prime}, \beta, \beta^{\prime}, \gamma^{\rho-1}} t_{\nu}(\lambda t)\right)(x)= & x^{\rho+\gamma-\alpha-\alpha^{\prime}-1} \frac{\Gamma(\nu+1)}{\sqrt{\pi}} \\
& \times{ }_{4} \Psi_{4}\left[\begin{array}{c}
\left(\frac{1}{2}, \frac{1}{2}\right),(\rho, 1),\left(\rho+\gamma-\alpha-\alpha^{\prime}-\beta, 1\right),\left(\rho+\beta^{\prime}-\alpha^{\prime}, 1\right) \\
\left(\nu+1, \frac{1}{2}\right),\left(\rho+\beta^{\prime}, 1\right),\left(\rho+\gamma-\alpha-\alpha^{\prime}, 1\right),\left(\rho+\gamma-\alpha^{\prime}-\beta, n\right)
\end{array} \mid \lambda x\right] .
\end{aligned}
$$

Proof. Applying (11) and using the definition (1) to (12), we get

$$
\begin{aligned}
& \left(I_{0,+}^{\alpha, \alpha^{\prime}, \beta, \beta^{\prime}, \gamma^{\rho}} t^{\rho-1} S_{\nu}(\lambda t)\right)(x) \\
& \quad=\left(I_{0,+}^{\alpha, \alpha^{\prime}, \beta, \beta^{\prime}, \gamma} \sum_{n=0}^{\infty} \frac{(\lambda)^{n} \Gamma(\nu+1) \Gamma((n+1) / 2)}{\sqrt{\pi} n ! \Gamma(n / 2+\nu+1)} t^{\rho+n-1}\right)
\end{aligned}
$$

$\cdot(x)$.

By changing the order of integration and summation,

$$
\begin{aligned}
& \left(I_{0,+}^{\alpha, \alpha^{\prime}, \beta, \beta^{\prime}, \gamma} t^{\rho-1} S_{\nu}(\lambda t)\right)(x) \\
& \quad=\sum_{n=0}^{\infty} \frac{(\lambda)^{n} \Gamma(\nu+1) \Gamma((n+1) / 2)}{\sqrt{\pi} n ! \Gamma(n / 2+\nu+1)}\left(I_{0,+}^{\alpha, \alpha^{\prime}, \beta, \beta^{\prime}, \gamma} t^{\rho+n-1}\right)
\end{aligned}
$$

$\cdot(x)$.
Hence replacing $\rho$ by $\rho+n$ in Lemma 1 , after some simplification, we obtain the following expression:

$$
\begin{aligned}
& \left(I_{0,+}^{\alpha, \alpha^{\prime}, \beta, \beta^{\prime}, \gamma} t^{\rho-1} S_{\nu}(\lambda t)\right)(x)=\sum_{n=0}^{\infty} \frac{\Gamma(\nu+1) \Gamma((n+1) / 2) \lambda^{n}}{\sqrt{\pi} n ! \Gamma(n / 2+\nu+1)} \\
& \cdot \frac{\Gamma(\rho+n) \Gamma\left(\rho+n+\gamma-\alpha-\alpha^{\prime}-\beta\right) \Gamma\left(\rho+n+\beta^{\prime}-\alpha^{\prime}\right)}{\Gamma\left(\rho+n+\beta^{\prime}\right) \Gamma\left(\rho+n+\gamma-\alpha-\alpha^{\prime}\right) \Gamma\left(\rho+n+\gamma-\alpha^{\prime}-\beta\right)} \\
& \cdot x^{\rho+n+\gamma-\alpha-\alpha^{\prime}-1}=\frac{x^{\rho+\gamma-\alpha-\alpha^{\prime}-1}}{\sqrt{\pi}} \Gamma(\nu+1) \sum_{n=0}^{\infty} \frac{\Gamma(n / 2+1 / 2)}{\Gamma(n / 2+\nu+1)} \\
& \cdot \frac{\Gamma(\rho+n) \Gamma\left(\rho+n+\gamma-\alpha-\alpha^{\prime}-\beta\right) \Gamma\left(\rho+n+\beta^{\prime}-\alpha^{\prime}\right)}{\Gamma\left(\rho+n+\beta^{\prime}\right) \Gamma\left(\rho+n+\gamma-\alpha-\alpha^{\prime}\right) \Gamma\left(\rho+n+\gamma-\alpha^{\prime}-\beta\right)} \\
& \cdot \frac{(x \lambda)^{n}}{n !}
\end{aligned}
$$

whose last summation, in view of (8), is easily seen to arrive at the expression (12).

Theorem 4. Let $\alpha, \alpha^{\prime}, \beta, \beta^{\prime}, \gamma, \rho, p, b, c, \lambda, \nu \in \mathbb{C}$. Suppose that $\operatorname{Re}(\gamma)>0$ and $\operatorname{Re}(\rho-n)<1+\min \left\{\operatorname{Re}(-\beta), \operatorname{Re}\left(\alpha+\alpha^{\prime}-\right.\right.$ $\left.\gamma), \operatorname{Re}\left(\alpha+\beta^{\prime}-\gamma\right)\right\}$. Then

$$
\begin{aligned}
& \left(I_{0,-}^{\alpha, \alpha^{\prime}, \beta, \beta^{\prime}, \gamma} t^{\rho-1} S_{\nu}\left(\frac{\lambda}{t}\right)\right)(x) \\
& =\frac{x^{\rho-\alpha-\alpha^{\prime}+\gamma-1}}{\sqrt{\pi}} \Gamma(\nu+1) \\
& \quad \times{ }_{4} \Psi_{4}\left[\begin{array}{c}
\left(\frac{1}{2}, \frac{1}{2}\right),\left(1-\rho-\gamma+\alpha+\alpha^{\prime}, 1\right),\left(1-\rho+\alpha+\beta^{\prime}, 1\right),\left(1-\rho-\beta^{\prime}, 1\right) \\
\left(\nu+1, \frac{1}{2}\right),(1-\rho, 1),\left(1-\rho+\alpha+\alpha^{\prime}+\beta+\beta^{\prime}-\gamma, 1\right),(1-\rho+n+\alpha+\beta, 1)
\end{array} \mid \lambda x\right] .
\end{aligned}
$$


Proof. Using (2) and (11) and then changing the order of integration and summation,

$$
\begin{aligned}
& \left(I_{0,-}^{\alpha, \alpha^{\prime}, \beta, \beta^{\prime}, \gamma} t^{\rho-1} S_{\nu}\left(\frac{\lambda}{t}\right)\right)(x) \\
& =\sum_{n=0}^{\infty} \frac{(\lambda)^{n} \Gamma(\nu+1) \Gamma((n+1) / 2)}{\sqrt{\pi} n ! \Gamma(n / 2+\nu+1)}\left(I_{0,-}^{\alpha, \alpha^{\prime}, \beta, \beta^{\prime}, \gamma} t^{\rho-n-1}\right) \\
& \quad(x) .
\end{aligned}
$$

Using Lemma 2, after a little simplification, we obtain the following expression:

$$
\begin{aligned}
& \left(I_{0,-}^{\alpha, \alpha^{\prime}, \beta, \beta^{\prime}, \gamma} t^{\rho-1} S_{\nu}\left(\frac{\lambda}{t}\right)\right)(x) \\
& \quad=\sum_{n=0}^{\infty} \frac{(\lambda)^{n} \Gamma(\nu+1) \Gamma((n+1) / 2)}{\sqrt{\pi} n ! \Gamma(n / 2+\nu+1)} \frac{\Gamma\left(1-\rho-n-\gamma+\alpha+\alpha^{\prime}\right) \Gamma\left(1-\rho-n+\alpha+\beta^{\prime}\right) \Gamma(1-\rho-n-\beta)}{\Gamma(1-\rho-n) \Gamma\left(1-\rho-n+\alpha+\alpha^{\prime}+\beta+\beta^{\prime}-\gamma\right) \Gamma(1-\rho-n+\alpha+\beta)} x^{\rho-n-\alpha-\alpha^{\prime}+\gamma-1} \\
& \quad=\frac{x^{\rho-\alpha-\alpha^{\prime}+\gamma-1}}{\sqrt{\pi}} \frac{\Gamma(\nu+1) \Gamma((n+1) / 2)}{\Gamma(n / 2+\nu+1)} \frac{\Gamma\left(1-\rho+n-\gamma+\alpha+\alpha^{\prime}\right) \Gamma\left(1-\rho+n+\alpha+\beta^{\prime}\right) \Gamma(1-\rho+n-\beta)}{\Gamma(1-\rho+n) \Gamma\left(1-\rho+n+\alpha+\alpha^{\prime}+\beta+\beta^{\prime}-\gamma\right) \Gamma(1-\rho+n+\alpha+\beta)} \frac{(x \lambda)^{n}}{n !} .
\end{aligned}
$$

In view of (8), we obtained the desired result (16).

2.1. Representation of Bessel-Struve Kernel Function in terms of Exponential Function. In this subsection we represent the Bessel-Struve function in terms of exponential function. Also, we derive the Marichev-Saigo-Maeda operator representation of these special cases. The representation of Bessel-Struve kernel function in terms of exponential function is

$$
\begin{aligned}
& S_{-1 / 2}(x)=e^{x}, \\
& S_{1 / 2}(x)=\frac{-1+e^{x}}{x} .
\end{aligned}
$$

Now, we have the following theorems.

Theorem 5. Let $\alpha, \alpha^{\prime}, \beta, \beta^{\prime}, \gamma, \rho, p, b, c, \lambda \in \mathbb{C}$. Suppose that $\operatorname{Re}(\gamma)>0$ and $\operatorname{Re}(\rho+n)>\max \left\{0, \operatorname{Re}\left(\alpha+\alpha^{\prime}+\beta-\gamma\right), \operatorname{Re}\left(\alpha^{\prime}-\right.\right.$ $\left.\left.\beta^{\prime}\right)\right\}$. Then

$$
\left(I_{0,+}^{\alpha, \alpha^{\prime}, \beta, \beta^{\prime}, \gamma} t^{\rho-1} e^{t}\right)(x)=x^{\rho-\alpha-\alpha^{\prime}+\gamma-1} \times{ }_{3} \Psi_{3}\left[\begin{array}{c}
(\rho, 1),\left(\rho+\gamma-\alpha-\alpha^{\prime}-\beta, 1\right),\left(\rho+\beta^{\prime}-\alpha^{\prime}, 1\right),\left(1-\rho-\beta^{\prime}, 1\right) \\
\left(\rho+\beta^{\prime}, 1\right),\left(\rho+\gamma-\alpha-\alpha^{\prime}, 1\right),\left(\rho+\gamma-\alpha^{\prime}-\beta, 1\right)
\end{array}\right] x .
$$

Proof. From (1), (19), and the definition of Bessel-Struve kernel function (11), we have

$$
\begin{aligned}
& \left(I_{0,+}^{\alpha, \alpha^{\prime}, \beta, \beta^{\prime}, \gamma} t^{\rho-1} e^{t}\right)(x) \\
& \quad=\left(I_{0,+}^{\alpha, \alpha^{\prime}, \beta, \beta^{\prime}, \gamma} t^{\rho-1} \sum_{n=0}^{\infty} \frac{\Gamma(-1 / 2+1) \Gamma((n+1) / 2)}{\sqrt{\pi} n ! \Gamma(n / 2-1 / 2+1)} t^{n}\right)
\end{aligned}
$$

$$
\begin{aligned}
& \cdot(x) \\
& =\sum_{n=0}^{\infty} \frac{\Gamma(1 / 2) \Gamma((n+1) / 2)}{\sqrt{\pi} n ! \Gamma((n+1) / 2)}\left(I_{0,+}^{\alpha, \alpha^{\prime}, \beta, \beta^{\prime}, \gamma} t^{\rho+n-1}\right)(x) .
\end{aligned}
$$

This together with Lemma 1 yields

$$
\left(I_{0,+}^{\alpha, \alpha^{\prime}, \beta, \beta^{\prime}, \gamma} t^{\rho-1} e^{t}\right)(x)=x^{\rho-\alpha-\alpha^{\prime}+\gamma-1} \sum_{n=0}^{\infty} \frac{x^{n}}{n !} \Gamma\left[\begin{array}{c}
\rho+n, \rho+n+\gamma-\alpha-\alpha^{\prime}-\beta, \rho+n+\beta^{\prime}-\alpha^{\prime} \\
\rho+n+\beta^{\prime}, \rho+n+\gamma-\alpha-\alpha^{\prime}, \rho+n+\gamma-\alpha^{\prime}-\beta
\end{array}\right]
$$
which is the desired result.

Theorem 6. Let $\alpha, \alpha^{\prime}, \beta, \beta^{\prime}, \gamma, \rho, p, b, c, \lambda \in \mathbb{C}$. Suppose that $\operatorname{Re}(\gamma)>0$ and $\operatorname{Re}(\rho+n)>\max \left\{0, \operatorname{Re}\left(\alpha+\alpha^{\prime}+\beta-\gamma\right), \operatorname{Re}\left(\alpha^{\prime}-\right.\right.$ 


$$
\begin{aligned}
\left(I_{0,+}^{\alpha, \alpha^{\prime}, \beta, \beta^{\prime}, \gamma} t^{\rho-1}\left(\frac{-1+e^{t}}{t}\right)\right)(x)= & x^{\rho-\alpha-\alpha^{\prime}+\gamma-1} \\
& \times{ }_{4} \Psi_{4}\left[\begin{array}{r}
\left(\frac{1}{2}, \frac{1}{2}\right),(\rho, 1),\left(\rho+\gamma-\alpha-\alpha^{\prime}-\beta, 1\right),\left(\rho+\beta^{\prime}-\alpha^{\prime}, 1\right) \\
\left.\left(\frac{1}{2}, \frac{3}{2}\right),\left(\rho+\beta^{\prime}, 1\right),\left(\rho+\gamma-\alpha-\alpha^{\prime}, 1\right),\left(\rho+\gamma-\alpha^{\prime}-\beta, 1\right) \mid x\right]
\end{array}\right] .
\end{aligned}
$$

Proof. The proof of the fractional integration formula (24) would run parallel to the proof of (21) by considering (20). Therefore, we omit the details.

2.2. Relation between Bessel-Struve Kernel Function and Bessel and Struve Function of First Kind. In this subsection we show the relation between $S_{\alpha}(x)$ and modified Bessel function $I_{v}(x)$ and modified Struve function $L_{v}(x)$ by choosing particular values of $\alpha$ :

$$
\begin{aligned}
& S_{0}(x)=I_{0}(x)+L_{0}(x), \\
& S_{1}(x)=\frac{2 I_{1}(x)+L_{1}(x)}{x} .
\end{aligned}
$$

In the coming two theorems, we give the fractional integral representations of (25) and (26).

Theorem 7. Let $\alpha, \alpha^{\prime}, \beta, \beta^{\prime}, \gamma, \rho, p, b, c, \lambda \in \mathbb{C}$. Suppose that $\operatorname{Re}(\gamma)>0$ and $\operatorname{Re}(\rho+n)>\max \left\{0, \operatorname{Re}\left(\alpha+\alpha^{\prime}+\beta-\gamma\right), \operatorname{Re}\left(\alpha^{\prime}-\right.\right.$ $\left.\left.\beta^{\prime}\right)\right\}$. Then

$$
\begin{aligned}
\left(I_{0,+}^{\alpha, \alpha^{\prime}, \beta, \beta^{\prime}, \gamma} t^{\rho-1}\left(I_{0}(t)+L_{0}(t)\right)\right)(x)= & \frac{x^{\rho-\alpha-\alpha^{\prime}+\gamma-1}}{\sqrt{\pi}} \\
& \times{ }_{4} \Psi_{4}\left[\begin{array}{r}
\left(\frac{1}{2}, \frac{1}{2}\right),(\rho, 1),\left(\rho+\gamma-\alpha-\alpha^{\prime}-\beta, 1\right),\left(\rho+\beta^{\prime}-\alpha^{\prime}, 1\right) \mid \\
\left(\frac{1}{2}, 1\right),\left(\rho+\beta^{\prime}, 1\right),\left(\rho+\gamma-\alpha-\alpha^{\prime}, 1\right),\left(\rho+\gamma-\alpha^{\prime}-\beta, 1\right) \mid x
\end{array}\right]
\end{aligned}
$$

Proof. Applying (26) and using the definition (1) to (27), we get

$$
\begin{aligned}
& \left(I_{0,+}^{\alpha, \alpha^{\prime}, \beta, \beta^{\prime}, \gamma} t_{0}^{\rho-1}\left(I_{0}(t)+L_{0}(t)\right)\right)(x) \\
& \quad=\left(I_{0,+}^{\alpha, \alpha^{\prime}, \beta, \beta^{\prime}, \gamma} t^{\rho-1} \sum_{n=0}^{\infty} \frac{\Gamma(1) \Gamma((n+1) / 2)}{\sqrt{\pi} n ! \Gamma(n / 2+1)} t^{n}\right)(x) \\
& \quad=\sum_{n=0}^{\infty} \frac{\Gamma((n+1) / 2)}{\sqrt{\pi} n ! \Gamma(n / 2+1)}\left(I_{0,+}^{\alpha, \alpha^{\prime}, \beta, \beta^{\prime}, \gamma} t^{\rho+n-1}\right)(x) .
\end{aligned}
$$

$$
\begin{aligned}
\left(I_{0,+}^{\alpha, \alpha^{\prime}, \beta, \beta^{\prime}, \gamma} t^{\rho-1} \frac{\left(2 I_{1}(t)+L_{1}(t)\right)}{t}\right)(x)= & \frac{x^{\rho-\alpha-\alpha^{\prime}+\gamma-1}}{\sqrt{\pi}} \\
& \times{ }_{4} \Psi_{4}\left[\begin{array}{c}
\left(\frac{1}{2}, \frac{1}{2}\right),(\rho, 1),\left(\rho+\gamma-\alpha-\alpha^{\prime}-\beta, 1\right),\left(\rho+\beta^{\prime}-\alpha^{\prime}, 1\right) \mid \\
\left(\frac{1}{2}, 1\right),\left(\rho+\beta^{\prime}, 1\right),\left(\rho+\gamma-\alpha-\alpha^{\prime}, 1\right),\left(\rho+\gamma-\alpha^{\prime}-\beta, 1\right) \mid x
\end{array}\right] .
\end{aligned}
$$


Proof. The details of the proof are omitted because it runs as parallel as the proof of (27).

\section{Pathway Fractional Integration of Bessel-Struve Kernel Function}

The pathway fractional integral operator was introduced and studied by Mathai [41] and Nair [42] and developed further by Mathai and Haubold $([43,44])$ as follows.

Let $f(x) \in L(a, b), \eta \in C, R(\eta)>0, a>0$ and the pathway parameter $\alpha<1$; then

$$
\begin{aligned}
& \left(P_{0+}^{(\eta, \alpha)} f\right)(x) \\
& \quad=x^{\eta} \int_{0}^{[x / a(1-\alpha)]} 1-\left[\frac{a(1-\alpha) t}{x}\right]^{\eta /(1-\alpha)} f(t) d t .
\end{aligned}
$$

For a real scalar $\alpha$, the pathway model for scalar random variables is represented by the following probability density function (p.d.f.):

$$
f(x)=c|x|^{\gamma-1}\left[1-a(1-\alpha)|x|^{\delta}\right]^{\beta /(1-\alpha)}
$$

provided that $-\infty<x<\infty, \delta>0, \beta \geq 0$, [1-a(1$\left.\alpha)|x|^{\delta}\right]>0$, and $\gamma>0$, where $c$ is the normalizing constant and $\alpha$ is called the pathway parameter (for details, see [42]).

Here, we investigate the composition formula of integral transform operator due to Nair, which is expressed in terms of the generalized Wright hypergeometric function, by inserting the generalized Bessel-Struve kernel $S_{\alpha}(\lambda z)$ which is defined in (11). The results given in this section are based on the preliminary assertions given by composition formula of pathway fractional integral (31) with a power function.

Lemma 9 (see [42]). Let $\eta \in C, \operatorname{Re}(\eta)>0, \beta \in C$, and $\alpha<1$. If $\operatorname{Re}(\beta)>0$ and $\operatorname{Re}(\eta /(1-\alpha))>-1$, then

$$
\begin{aligned}
& \left\{P_{0+}^{(\eta, \alpha)}\left[t^{\beta-1}\right]\right\}(x) \\
& \quad=\frac{x^{\eta+\beta}}{[a(1-\alpha)]^{\beta}} \frac{\Gamma(\beta) \Gamma(1+\eta /(1-\alpha))}{\Gamma(1+\eta /(1-\alpha)+\beta)} .
\end{aligned}
$$

Now, we have the following theorems.

Theorem 10. Let $\eta, \sigma, p, b, c, \lambda \in \mathbb{C}$ and $\alpha<1$ such that $\operatorname{Re}(\eta)>0, \operatorname{Re}(\sigma)>0, \operatorname{Re}(\sigma+n)>0$, and $\operatorname{Re}(\eta /(1-\alpha))>-1 ;$ then the following formula holds:

$$
\begin{aligned}
& \left(P_{0+}^{(\eta, \alpha)}\left[t^{\sigma-1} S_{\nu}(\lambda t)\right]\right)(x) \\
& =x^{\eta+\sigma} \frac{\Gamma(\nu+1) \mu(1+\eta /(1-\alpha))}{\sqrt{\pi}[a(1-\alpha)]^{\sigma+p+1}} \\
& \quad \times{ }_{2} \Psi_{2}\left[\begin{array}{c}
\left(\frac{1}{2}, \frac{1}{2}\right),(\rho, 1) \\
\left(\nu+1, \frac{1}{2}\right),\left(1+\frac{\eta}{1-\alpha}+\sigma, 1\right)
\end{array} ; \lambda x\right] .
\end{aligned}
$$

Proof. Applying (11) and (31) and changing the order of integration and summation, we get

$$
\begin{aligned}
& \left(P_{0+}^{(\eta, \alpha)}\left[t^{\sigma-1} S_{\nu}(\lambda t)\right]\right)(x) \\
& =\left(P_{0+}^{(\eta, \alpha)}\left[t^{\sigma-1} \sum_{n=0}^{\infty} \frac{\lambda^{n} \Gamma(\alpha+1) \Gamma((n+1) / 2)}{\sqrt{\pi} n ! \Gamma(n / 2+\alpha+1)} t^{n}\right]\right)(x) \\
& =\sum_{k=0}^{\infty} \frac{\lambda^{n} \Gamma(\nu+1) \Gamma((n+1) / 2)}{\sqrt{\pi} n ! \Gamma(n / 2+\nu+1)}\left(P_{0+}^{(\eta, \alpha)}\left\{t^{(n+\sigma)-1}\right\}\right)(x) .
\end{aligned}
$$

Using the conditions mentioned in the statement of the theorem and $k \in K_{0}, R(p+n)>0, \operatorname{Re}(\eta /(1-\alpha))>-1$. Applying Lemma 9 and using (33) with $\beta$ replaced by $\sigma+n$, we get

$$
\begin{aligned}
\left(P_{0+}^{(\eta, \alpha)}\left[t^{\sigma-1} S_{\nu}(\lambda t)\right]\right)(x) \\
=\sum_{k=0}^{\infty} \frac{\lambda^{n} \Gamma(\nu+1) \Gamma((n+1) / 2)}{\sqrt{\pi} n ! \Gamma(n / 2+\nu+1)} \frac{x^{\eta+\alpha}}{\left[a(1-\alpha)^{\sigma+n}\right]} \\
\quad \cdot \frac{\Gamma(\sigma+n) \Gamma(1+\eta /(1-\alpha))}{\Gamma(1+\eta /(1-\alpha)+\sigma+n)} \\
=\frac{x^{\eta+\alpha} \Gamma(\nu+1) \Gamma(1+\eta /(1-\alpha))}{\sqrt{\pi}\left[a(1-\alpha)^{\sigma}\right]} \\
\quad \cdot \sum_{k=0}^{\infty} \frac{\Gamma(n / 2+1 / 2) \Gamma(\sigma+n)}{\Gamma(n / 2+\nu+1) \Gamma(1+\eta /(1-\alpha)+\sigma+n)} \frac{(x \lambda)^{n}}{n !}
\end{aligned}
$$

which gives the desired result.

By considering the relations given in (19) and (20), we obtain various new integral formulas for Bessel-Struve functions involved in the pathway fractional integration operators.

Theorem 11. Let $\eta, \sigma, p, b, c \in C$ and $\alpha<1$ such that $\operatorname{Re}(\eta)>$ $0, \operatorname{Re}(\sigma+n)>0$, and $\operatorname{Re}(\eta /(1-\alpha))>-1$; then the following formula holds:

$$
\begin{aligned}
& \left(P_{0+}^{(\eta, \alpha)}\left[t^{\sigma-1} e^{t}\right]\right)(x) \\
& =x^{\eta+\sigma} \frac{\Gamma(1+\eta /(1-\alpha))}{[a(1-\alpha)]^{\sigma}} \\
& \times{ }_{1} \Psi_{1}\left[\left(1+\sigma+\frac{\eta}{1-\alpha}, 1\right) \mid \frac{x}{[a(1-\alpha)]}\right] .
\end{aligned}
$$

Proof. Applying (19) and using (31) with the help of Lemma 9 we can easily prove (37), so the details are omitted. 
Theorem 12. Let $\eta, \sigma, p, b, c \in C$ and $\alpha<1$ such that $\operatorname{Re}(\sigma+$ $n)>0$ and $\operatorname{Re}(\eta /(1-\alpha))>-1$; then the following formula holds:

$$
\begin{aligned}
& \left(P_{0+}^{(\eta, \alpha)}\left[t^{\sigma-1} \frac{-1+e^{t}}{t}\right]\right)(x)=x^{\eta+\sigma} \frac{\Gamma(1+\eta /(1-\alpha))}{2[a(1-\alpha)]^{\sigma}}
\end{aligned}
$$

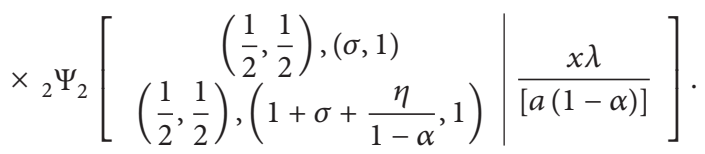

Proof. The details of proof are omitted because the result (38) can easily derive as similar as the procedure of the proof of Theorem 10 using (20), (11), and (31).

Remark 13. As similar as the method discussed in Theorem 10, one can easily derive the pathway integral representation of (25) and (26).

\section{Conclusion}

Fractional integral formulas involving Bessel-Struve kernel function $S_{\alpha}(\lambda z), \lambda, z \in \mathbb{C}$ have been developed and studied in this paper. The pathway integral representations BesselStruve kernel function and its relation between many other functions are also derived in this study. The results thus given in this paper are general in character and likely to find some applications in the theory of special functions.

\section{Competing Interests}

The authors declare that they have no competing interests.

\section{Authors' Contributions}

All authors have equal contributions. All authors read and approved the final paper.

\section{Acknowledgments}

This project was supported by the Deanship of Scientific Research at Prince Sattam Bin Abdulaziz University under the research project \#2015/01/3883.

\section{References}

[1] D. Valerio and J. S. Costa, "Introduction to single-input, singleoutput fractional control," IET Control Theory \& Applications, vol. 5, no. 8, pp. 1033-1057, 2011.

[2] Y. Jin, Y.-Q. Chen, and D. Xue, "Time-constant robust analysis of a fractional order [proportional derivative] controller," IET Control Theory and Applications, vol. 5, no. 1, pp. 164-172, 2011.

[3] I. Podlubny, Fractional-Order Systems and Fractional-Order Controllers, UEF-03-94, Institute of Experimental Physics, Slovak Academy of Sciences, Kosice, Slovakia, 1994.

[4] R. Caponetto, G. Dongola, and I. Petras, Fractional Order Systems, Modeling and Control Applications, vol. 72 of World Scientific A, 2010.
[5] D. Baleanu, "About fractional quantization and fractional variational principles," Communications in Nonlinear Science and Numerical Simulation, vol. 14, no. 6, pp. 2520-2523, 2009.

[6] V. Kiryakova, Generalized Fractional Calculus and Applications, vol. 301, Longman Scientific \& Technical, Essex, UK, 1994.

[7] D. Băleanu and O. G. Mustafa, "On the global existence of solutions to a class of fractional differential equations," Computers \& Mathematics with Applications, vol. 59, no. 5, pp. 1835-1841, 2010.

[8] E. R. Love, "Some integral equations involving hypergeometric functions," Proceedings of the Edinburgh Mathematical Society, vol. 15, no. 3, pp. 169-198, 1967.

[9] A. C. McBride, "Fractional powers of a class of ordinary differentilal operators," Proceedings of the London Mathematical Society, vol. 45, no. 3, pp. 519-546, 1982.

[10] P. Agarwal and J. J. Nieto, "Some fractional integral formulas for the Mittag-Leffler type function with four parameters," Open Mathematics, vol. 13, no. 1, pp. 537-546, 2015.

[11] S. L. Kalla, "Integral operators involving Fox's H-function II," Acta Mexicana de Ciencia y Tecnología, vol. 7, pp. 72-79, 1969.

[12] S. L. Kalla and R. K. Saxena, "Integral operators involving hypergeometric functions," Mathematische Zeitschrift, vol. 108, pp. 231-234, 1969.

[13] S. L. Kalla and R. K. Saxena, "Integral operators involving hypergeometric functions II," Universidad Nacional de Tucumán Revista Serie A: Matemática y Física Teórica, vol. 24, pp. 31-36, 1974.

[14] M. Saigo, "A remark on integral operators involving the Gauss hypergeometric functions," Mathematical Reports, College of General Education, Kyushu University, vol. 11, pp. 135-143, 1978.

[15] M. Saigo, "A certain boundary value problem for the EulerDarboux equation," Mathematica Japonica, vol. 24, no. 4, pp. 377-385, 1979.

[16] M. Saigo, "A certain boundary value problem for the EulerDarboux equation II," Mathematica Japonica, vol. 25, no. 2, pp. 211-220, 1980.

[17] M. Saigo and N. Maeda, "More generalization of fractional calculus," in Proceedings of the 2nd International Workshop on Transform Methods \& Special Functions, pp. 386-400, Varna, Bulgaria, August 1996.

[18] V. Kiryakova, Generalized Fractional Calculus and Applications, Longman Scientific \& Technical, Essex, UK, 1994.

[19] K. S. Miller and B. Ross, An Introduction to the Fractional Calculus and Differential Equations, John Wiley \& Sons, New York, NY, USA, 1993.

[20] A. A. Kilbas and N. Sebastian, "Generalized fractional integration of Bessel function of the first kind," Integral Transforms and Special Functions, vol. 19, no. 11-12, pp. 869-883, 2008.

[21] H. M. Srivastava and P. Agarwal, "Certain fractional integral operators and the generalized incomplete hypergeometric functions," Applications and Applied Mathematics, vol. 8, no. 2, pp. 333-345, 2013.

[22] S. R. Mondal and K. S. Nisar, "Marichev-Saigo-Maeda fractional integration operators involving generalized Bessel functions," Mathematical Problems in Engineering, vol. 2014, Article ID 274093, 11 pages, 2014.

[23] D. Baleanu and P. Agarwal, "On generalized fractional integral operators and the generalized Gauss hypergeometric functions," Abstract and Applied Analysis, vol. 2014, Article ID 630840, 5 pages, 2014. 
[24] O. I. Marichev, "Volterra equation of Mellin convolution type with a Horn function in the kernel," Izvestiya Akademii Nauk BSSR, Seriya Fiziko-Matematicheskikh Nauk, vol. 1, pp. 128-129, 1974 (Russian).

[25] S. Samko, A. Kilbas, and O. I. Marichev, Fractional Integrals and Derivatives, Theory and Applications, Gordon \& Breach Science Publishers, New York, NY, USA, 1993.

[26] H. M. Srivastava and P. W. Karlson, Multiple Gaussian Hypergeometric Series, Ellis Horwood Limited, New York, NY, USA, 1985.

[27] A. Erdélyi, W. Magnus, F. Oberhettinger, and F. G. Tricomi, Higher Transcendental Functions, vol. 1, McGraw-Hill, New York, NY, USA, 1953.

[28] A. P. Prudnikov, Yu. A. Brychkov, and O. I. Marichev, Integrals and Series. Special Functions, Vol. 1-5, Gordon \& Breach, New York, NY, USA, 1986.

[29] Y. C. Kim, K. S. Lee, and H. M. Srivastava, "Some applications of fractional integral operators and Ruscheweyh derivatives," Journal of Mathematical Analysis and Applications, vol. 197, no. 2, pp. 505-517, 1996.

[30] V. Kiryakova, "All the special functions are fractional differintegrals of elementary functions," Journal of Physics A: Mathematical and General, vol. 30, no. 14, pp. 5085-5103, 1997.

[31] H. M. Srivastava, S.-D. Lin, and P.-Y. Wang, "Some fractionalcalculus results for the $\bar{H}$-function associated with a class of Feynman integrals," Russian Journal of Mathematical Physics, vol. 13, no. 1, pp. 94-100, 2006.

[32] R. K. Saxena and M. Saigo, "Generalized fractional calculus of the $H$-function associated with the Appell function F3," Journal of Fractional Calculus, vol. 19, pp. 89-104, 2001.

[33] C. Fox, "The asymptotic expansion of generalized hypergeometric functions," Proceedings London Mathematical Society, vol. 27, no. 4, pp. 389-400, 1928.

[34] E. M. Wright, "The asymptotic expansion of integral functions defined by Taylor series," Philosophical Transactions of the Royal Society of London. Series A, vol. 238, pp. 423-451, 1940.

[35] E. M. Wright, "The asymptotic expansion of the generalized hypergeometric function," Proceedings of the London Mathematical Society, vol. 46, no. 2, pp. 389-408, 1940.

[36] A. A. Kilbas, M. Saigo, and J. J. Trujillo, "On the generalized Wright function," Fractional Calculus \& Applied Analysis, vol. 5, no. 4, pp. 437-460, 2002.

[37] A. A. Kilbas and N. Sebastian, "Fractional integration of the product of Bessel functions of the first kind," Fractional Calculus \& Applied Analysis, vol. 13, no. 2, pp. 159-175, 2010.

[38] H. M. Srivastava, "Some Fox-Wright generalized hypergeometric functions and associated families of convolution operators," Applicable Analysis and Discrete Mathematics, vol. 1, no. 1, pp. 56-71, 2007.

[39] G. N. Watson, A Treatise on the Theory of Bessel Functions, Cambridge University Press, Cambridge, UK, 1992.

[40] A. Gasmi and M. Sifi, "The Bessel-Struve intertwining operator on $\mathbb{C}$ and mean-periodic functions," International Journal of Mathematics and Mathematical Sciences, vol. 2004, no. 59, pp. 3171-3185, 2004.

[41] A. M. Mathai, "A pathway to matrix-variate gamma and normal densities," Linear Algebra and its Applications, vol. 396, pp. 317328, 2005.

[42] S. S. Nair, "Pathway fractional integration operator," Fractional Calculus \& Applied Analysis, vol. 12, no. 3, pp. 237-252, 2009.
[43] A. M. Mathai and H. J. Haubold, "On generalized distributions and pathways," Physics Letters A, vol. 372, no. 12, pp. 2109-2113, 2008.

[44] A. M. Mathai and H. J. Haubold, "Pathway model, superstatistics, Tsallis statistics, and a generalized measure of entropy," Physica A: Statistical Mechanics and Its Applications, vol. 375, no. 1, pp. 110-122, 2007. 


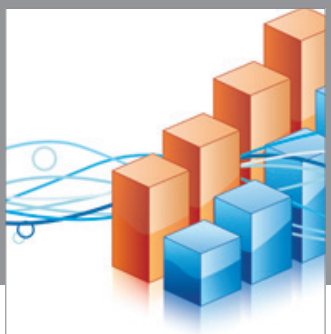

Advances in

Operations Research

vatem alat4

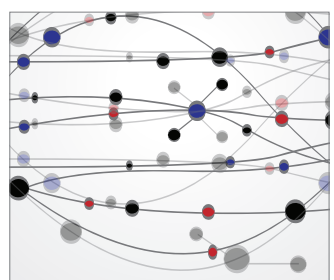

\section{The Scientific} World Journal
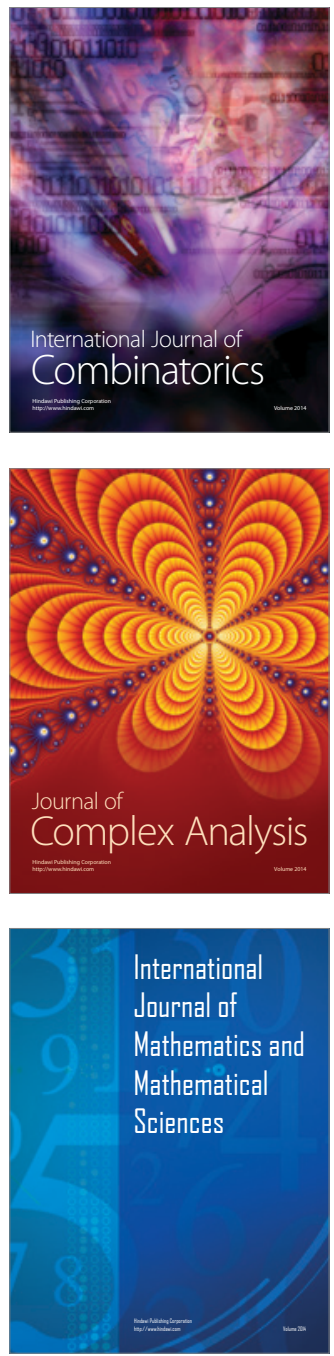
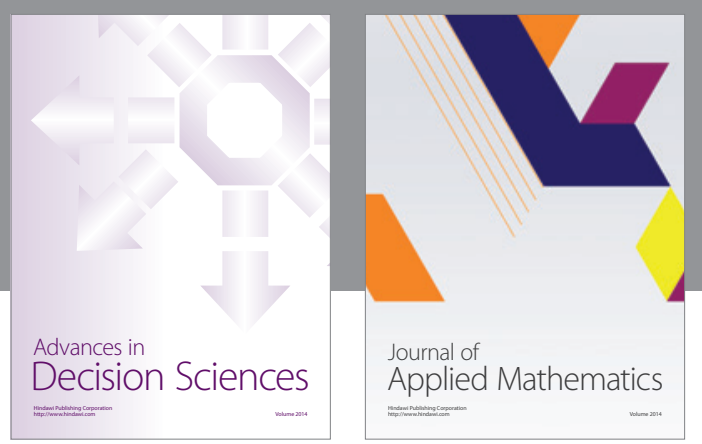

Algebra

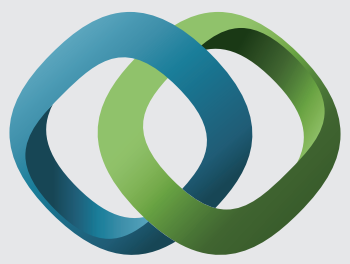

\section{Hindawi}

Submit your manuscripts at

http://www.hindawi.com
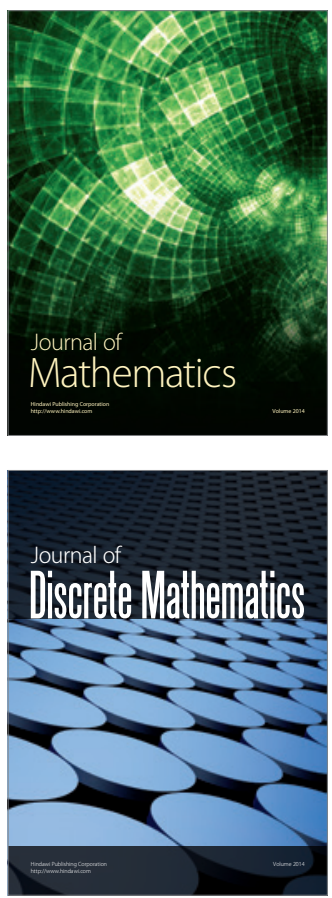

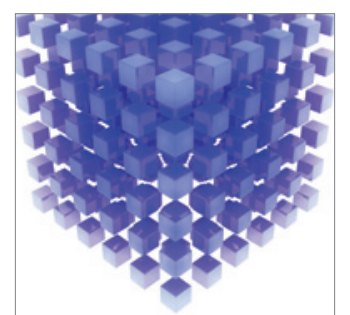

Mathematical Problems in Engineering
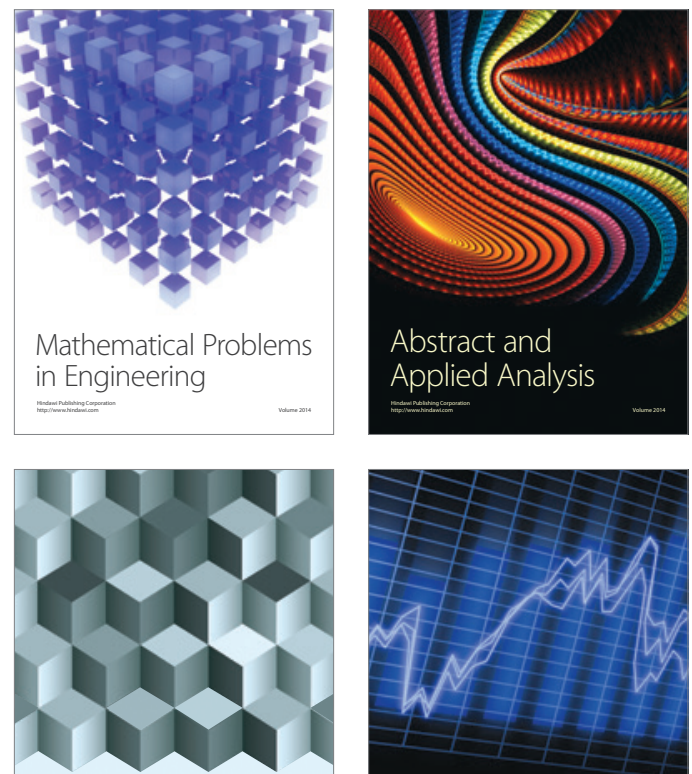

Journal of

Function Spaces

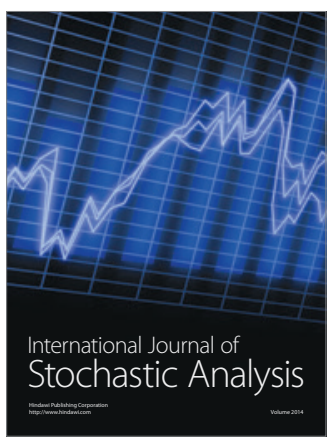

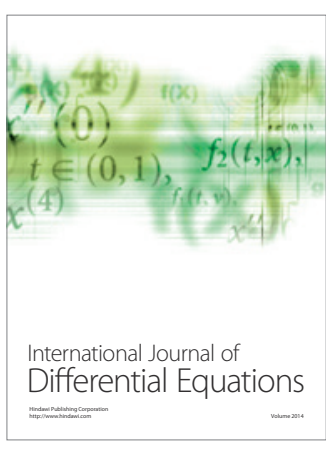
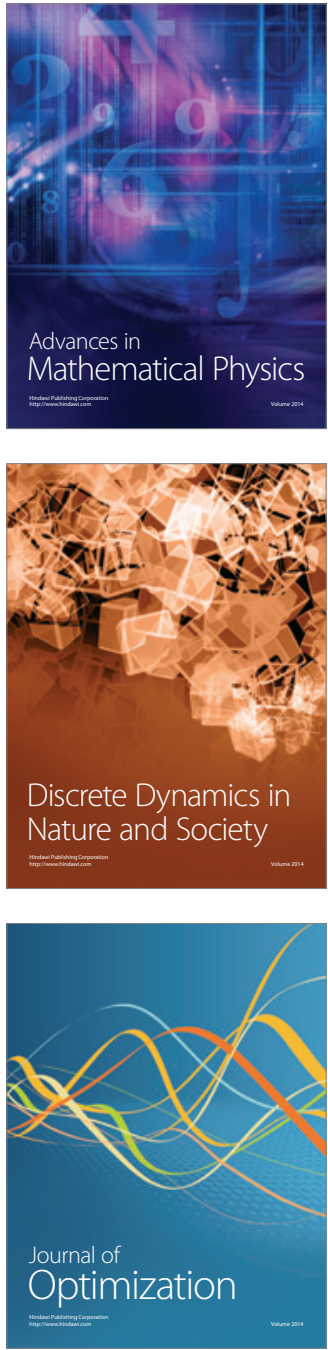